\title{
Conferencing in 2022
}

\author{
As the end of 2021 approaches, Nature Aging's editorial team reflects on our experience in the last two years of \\ conferencing and introduces a calendar for conferences on aging and age-related diseases for 2022.
}

T:

he COVID-19 pandemic has kept international researchers at a distance from each other for almost two years. Back in early 2020, countless conferences were cancelled. Soon followed a progressive transition to an all-'virtual' world and remote conferences became the norm. This helped to maintain the verbal sharing of knowledge and exchange of ideas that are essential to scientific research.

The virtual conferencing transition came with both pros and cons. On the plus side, conferences became easier to organize and attend, cheaper, more diverse and more environmentally friendly. Travel was taken out of the equation, leading to a reduction in meetings' carbon footprints, as well as less physical and mental strain and reduced time commitment for participants. Without the need for venues, conference fees were discounted or waived altogether, and without travel or accommodation, the overall cost of attendance was reduced by at least tenfold. This greatly lowered the barrier to accessing international meetings for all, and in particular for scientists from low- and middle-income countries.

On the negative side, we have all experienced the limitations of virtual conference platforms, such as the frequent absence of poster sessions, not being able to see who you speak to during a Q\&A, the sometimes-awkward moments in randomized and timed break-out rooms, and the absence of applause after talks. Networking, a crucial aspect of conferences, has also been a lot more difficult. In a recent Nature poll (A. Remmel, Nature 591, 185$186 ; 2021), 69 \%$ of respondents thought that remote conferences did not feature adequate networking opportunities. Not traveling overseas has also often meant continuing to live in our own time zones, thereby further limiting opportunities for interactions. In truth, many of these issues can be addressed by new platforms, technologies and individual efforts. But what remains the biggest issue with remote conferences is the absence of a true social dimension, one that exists only in physical proximity and that is essential to the development of human relationships. There is little spontaneity in our interactions at remote meetings. We do not bump into or get introduced to new people who may share the same interests. Visual social cues are mostly absent, and there are no handshakes or small talk to break the ice. Virtually, we cannot share a meal or a glass of our favorite beverage with our peers in a socially meaningful way. In other words, remote conferences feel disconnected and non-social, making them less enjoyable and less prone to fostering strong professional bonds or spurring collaborations than their in-person counterparts.

The good news is that it appears we are slowly returning to a world where in-person conferences are going to be possible again, and many of us are already rejoicing at the prospect of connecting or reconnecting with professional friends and colleagues in the real world. During this reverse transition, however, it is important to remember the positive aspects of remote conferences and it seems reasonable to say that, going forward, a hybrid world where remote and in-person experiences co-exist, sometimes at the same conferences, will be a better world.

As Nature Aging is looking forward to the return of in-person meetings in 2022, we are publishing today on our website an editorially curated calendar of conferences on aging and age-related diseases that we think will be relevant and useful to many of our readers. The list is non-exhaustive and we welcome suggestions. We will aim to update it regularly, beyond 2022, and will include information on whether the conferences are remote, in person or hybrid, and whether one of our editors will attend the meeting.

Some may wonder what editors do at conferences and how we decide which conferences to attend. Nature Aging editors typically participate in four or five conferences in a calendar year, which is a small number considering the extent of the journal's scope and the size of our team. We therefore aim for quality and a diversity of topics. An important goal for our editors is to invite authors to submit some of their best work to the journal. This is why we strongly favor meetings where organizers encourage the presentation of unpublished studies. We also expect to get up to speed with the latest developments in the field, connect with researchers at various career stages, get feedback on the journal, and discuss our policies and editorial practices. We often share our expertise in publishing or discuss publishing as a trade with students, postdocs and other earlycareer researchers during dedicated Q\&A sessions or informal conversations. We are also sometimes invited to contribute to panel discussions and it is important to mention that, to support gender diversity in science, we will participate only in events that are not dominated by male participants. Similarly, if asked to suggest speakers, we will strongly encourage organizers to consider the representation of minorities and geographical diversity.

Also on the theme of conferences, we announce that, starting in this issue (see here), the journal will occasionally publish Meeting Reports. These short articles present a selection of the most interesting work and ideas presented at a conference and produce a synthesis of what emerged from the meeting, discussing new trends and directions or important gaps in knowledge to be filled by future work. Meeting Reports must be discussed with our editors prior to submission, ideally before or at the time of the meeting in question.

If you are organizing a conference that is not on our list, please do not hesitate to suggest it as an addition (aging@nature.com) and we will carefully consider your request. We also hope to organize our very own Nature conference in the future. If you are interested in the possibility of co-organizing a meeting with us, please feel free to send us an expression of interest. We hope that our conference calendar will be a useful resource to our readers and look forward to meeting many of you in person at conferences in the coming year.

Published online: 22 December 2021 https://doi.org/10.1038/s43587-021-00157-w 